\title{
Suppression of tumor energy supply by liposomal nanoparticle-mediated inhibition of aerobic glycolysis
}

Yinlong Zhang ${ }^{1,2}$, Jingyan Wei ${ }^{1 *}$, Jiaqi $\mathrm{Xu}^{2}$, Wei Sun Leong ${ }^{3}$, Guangna Liu ${ }^{1,2}$, Tianjiao $\mathrm{Ji}^{2}$, Zhiqiang Cheng ${ }^{2}$, Jing Wang ${ }^{2}$, Jiayan Lang ${ }^{2}$, Ying Zhao ${ }^{2}$, Linhao $\mathrm{You}^{2}$, Xiao Zhao ${ }^{2}$, Taotao $\mathrm{Wei}^{4}$, Greg J Anderson ${ }^{5}$, Sheng $\mathrm{Qi}^{6}$, Jing Kong ${ }^{3}$, Guangjun $\mathrm{Nie}^{2,7 *}$, Suping $\mathrm{Li}^{2 *}$

${ }^{1}$ College of Pharmaceutical Science, Jilin University, Changchun 130021, China

${ }^{2}$ CAS Key Laboratory for Biomedical Effects of Nanomaterials \& Nanosafety, CAS Excellent Center for Nanoscience, National Center for Nanoscience and Technology, China, Beijing 100190, China

${ }^{3}$ Department of Electrical Engineering and Computer Science, Massachusetts Institute of Technology, Cambridge, MA 02139, USA

${ }^{4}$ National Laboratory of Biomacromolecules, Institute of Biophysics, Chinese Academy of Sciences, Beijing 100101, China

${ }^{5}$ QIMR Berghofer Medical Research Institute, Royal Brisbane Hospital, QLD 4029, Australia

${ }^{6}$ School of Pharmacy, University of East Anglia, Norwich, Norfolk NR4 7TJ, UK

${ }^{7}$ University of Chinese Academy of Sciences, Beijing 100049, China

*Corresponding authors:

Suping Li, email: lisuping@ nanoctr.cn;_Guangjun Nie, email: niegi@nanoctr.cn Jingyan Wei, email:jingyanweijluedu@163.com 


\begin{abstract}
Aerobic glycolysis enables cancer cells to rapidly take up nutrients (e.g., nucleotides, amino acids, lipids) and incorporate them into the biomass needed to produce a new cell. In contrast to existing chemotherapy/radiotherapy strategies, inhibiting aerobic glycolysis to limit ATP yield is a highly efficient approach for suppressing tumor cell proliferation. However, most, if not all, current inhibitors of aerobic glycolysis cause significant adverse effects, due to their nonspecific delivery and distribution to non-diseased organs, low bioavailability and a narrow therapeutic window. New strategies to enhance the biosafety and efficacy of these inhibitors are needed for moving them into clinical application. To address this need, we developed a liposomal nanocarrier functionalized with a well-validated tumor targeting peptide to specifically deliver the aerobic glycolysis inhibitor 3-bromopyruvate (3-BP) into tumor tissue. The nanoparticles effectively targeted tumors after systemic administration into tumor-bearing mice, and suppressed tumor growth by locally releasing 3-BP to inhibit ATP production of the tumor cells. No overt side effects were observed in the major organs. This report demonstrates the potential utility of the nanoparticle-enabled delivery of an aerobic glycolysis inhibitor as an anticancer therapeutic agent.
\end{abstract}

Key words: Warburg effect, ATP, 3-bromopyruvate, liposomal nanoparticles, tumor targeting peptide

\title{
1. Introduction
}

Unlike normal differentiated cells that rely primarily on mitochondrial oxidative phosphorylation to generate the adenosine $5^{\prime}$-triphosphate (ATP) needed for cellular processes ${ }^{1,2}$, tumor cells rely on aerobic glycolysis, a phenomenon termed the "Warburg effect." 3-5. Aerobic glycolysis is an inefficient way to generate ATP, however, it facilitates the uptake and incorporation of nutrients needed for tumor cell proliferation, such as nucleotides, amino acids, and lipids, into the biomass ${ }^{4}$. There is 
growing evidence that inhibiting aerobic glycolysis can directly induce tumor cell apoptosis through reducing ATP levels. The pyruvate mimetic 3-bromopyruvate (3-BP) is considered to be an effective inhibitor of glycolysis ${ }^{6,7}$, and has shown remarkable efficacy in inducing tumor cell apoptosis and preventing tumor growth in preclinical studies ${ }^{8-11}$. 3-BP is taken up into tumor cells via monocarboxylate transporter 1 (MCT1) which is overexpressed in most tumor cells, especially in hypoxic tumor regions ${ }^{12-14}$. Despite 3-BP showing considerable therapeutic potential against tumors, significant side effects, mainly owing to nonspecific delivery, distribution to non-diseased organs, low bioavailability and narrow therapeutic window, limit its clinical application ${ }^{15}$.

For in vivo use, the ideal scenario would be targeted delivery of therapeutic 3-BP to cancer cells that have particularly high expression of MCT1, thereby limiting side effects that result from nonspecific delivery as well as reducing the therapeutic dosage. Motivated by this rationale, we constructed biodegradable and biocompatible liposomal nanoparticles for the targeted delivery of 3-BP agent to tumors (Figure 1). We chose US Food and Drug Administration (FDA)-approved liposome components to construct the nanoparticles, thereby facilitating the translation of this nanovehicle into clinical practice ${ }^{16-19}$. We encapsulated 3-BP into nanoparticles using a filming-rehydration method, and covalently coupled a tumor targeting pentapeptide (Cys-Arg-Glu-Lys-Ala; CREKA) to their surface. CREKA recognizes fibrin-fibronectin complexes (microthrombi) which are specific for the tumor vascular endothelium, enabling the peptide-linked nanoparticles to accumulate at the site of the tumor in a self-amplifying manner ${ }^{20,21}$. The resulting nanoformulation (T-Lipo-3BP) effectively targeted to tumor vessels following systemic administration and released 3-BP to selectively kill tumor cells by blocking ATP production (Figure 1). This approach not only inhibited the growth of the tumor, but showed no or minimal side effects, unlike free 3-BP. Our strategy allows 3-BP to be delivered safely and more effectively than free drug, and paves the way for its potential clinical usage. 


\section{Materials and Methods}

\section{Materials}

1,2-distearoyl-sn-glycero-3-phosphoethanolamine- $\mathrm{N}$-[methoxy(polyethylene glycol)-2000] (DSPE-PEG-2000)

and 1,2-distearoyl-sn-glycero-3-phosphoethanolamine- $\mathrm{N}$-[maleimide(polyethylene glycol)-3400] (DSPE-PEG-3400-Mal) were purchased from Nuodepaisen Medical Technology Co. Ltd. (Suzhou, China). The peptide CREKA was synthesized by Taopu Biotechnology Co. Ltd (Shanghai, China). 3-Bromopyruvate (3-BP) was purchased from Beijing Inoke Technology Co. Ltd (Beijing, China). Cell counting kit-8 was purchased from Dojindo Molecular Technologies (Tokyo, Japan). Anti-Monocarboxylic acid transporter 1 antibody (Abcam: ab90582) and anti-HIF-1-alpha antibody (Abcam: ab16066) were obtained from Youningwei Biotechnology Co., Ltd (Shanghai, China).

\subsection{Synthesis of DSPE-PEG-CREKA}

DSPE-PEG-CREKA was prepared according to methods described in our previous work ${ }^{22}$. Briefly, $8.5 \mathrm{mg}$ DSPE-PEG-MAL and $1.78 \mathrm{mg}$ CREKA peptide were dissolved in $4 \mathrm{~mL}$ of $10 \%$ methanol and stirred for $24 \mathrm{~h}$ at room temperature under anaerobic conditions. The solution was then dialyzed (molecular weight cutoff of $\mathrm{M}_{\mathrm{r}}$ 1000) against tri-distilled water for $24 \mathrm{~h}$ and lyophilized for $48 \mathrm{~h}$.

\subsection{Preparation and characterization of nanoparticles}

T-Lipo-3BP and T-Lipo were prepared by a modified filming-rehydration method ${ }^{22}$. In brief, $20 \mathrm{mg}$ of lecithin, $2.36 \mathrm{mg}$ of DSPE-PEG-2000, $2.36 \mathrm{mg}$ of DSPE-PEG-CREKA and $4.9 \mathrm{mg}$ of cholesterol were dissolved in $10 \mathrm{~mL}$ of dichloromethane in a round-bottom flask. After rotary evaporation for $40 \mathrm{~min}$, a thin transparent film was formed. Phosphate-buffered saline (PBS) $(10 \mathrm{~mL})$ with varying amounts of 3-BP was added, and hydration was carried out for $20 \mathrm{~min}$ at $50^{\circ} \mathrm{C}$. The final product was filtered using a $200 \mu \mathrm{m}$ liposome extruder. Free 3-BP was then removed by 24 h of ultrafiltration (MWCO: 2000). The final solution was collected and used for characterization of the nanoparticles, drug release profile tests and in vivo experiments. For in vivo imaging, 3-BP was replaced with $\mathrm{Cy} 7$ fluorescent dye 
during the synthetic process. The size distribution and zeta potential of T-Lipo and T-Lipo-3BP were assessed by dynamic light scattering (DLS) using a ZetaSizer Nano series Nano-ZS (Malvern Instruments Ltd., Malvern, UK). The morphology of T-Lipo-3BP and T-Lipo was characterized using transmission electron microscopy (TEM) with a negative staining method as previously described ${ }^{22}$ and using a EM-200CX electron microscope (JEOL Ltd., Tokyo, Japan).

\subsection{In vitro drug release profile of T-Lipo-3BP}

The in vitro drug release kinetic profile of T-Lipo-3BP was evaluated by dialysis. Briefly, $2 \mathrm{~mL}$ of T-Lipo-3BP was added to a dialysis bag (molecular weight cutoff of $\mathrm{M}_{\mathrm{r}}$ 1000) and placed into $40 \mathrm{~mL}$ of $\mathrm{PBS}$ at $\mathrm{pH} 7.4$ or $\mathrm{pH} 6.8$ in a $50 \mathrm{~mL}$ tube. The tube was shaken at $37^{\circ} \mathrm{C}$. At various times, $1 \mathrm{~mL}$ of the outside buffer was removed and replaced with $1 \mathrm{~mL}$ of fresh PBS. The amount of 3-BP contained in the PBS was assayed using high-performance liquid chromatography (HPLC). In brief, a C18-alkyl reverse phase bonded column was chosen as the stationary phase. The mobile phase contained a $90 / 10$ mixture of $0.1 \%$ trifluoroacetic acid in water/0.1\% trifluoroacetic acid in acetonitrile. An isocratic elution method with a flow rate of $1 \mathrm{~mL}$ per minute and a run time of 5 minutes was used. 3-BP levels were assessed by measuring the absorbance using an ultraviolet detector at $204 \mathrm{~nm}$. A standard curve for 3-BP was generated using drug concentrations of $0.1875,0.375,0.75,1.5$ and $3 \mathrm{mg} / \mathrm{mL}$. Samples were analyzed in triplicate and solvent alone was used as the blank.

\subsection{Cytotoxicity assays}

The mouse pancreatic cancer cell line Pan-02 was kindly supplied by Professor H. Lee from Moffitt Cancer Center and Research Institute, Tampa, USA, and maintained at $37^{\circ} \mathrm{C}$ and $5 \% \mathrm{CO}_{2}$ in RPMI1640 medium supplemented with $10 \%$ Fetal Bovine Serum (FBS) and 1\% penicillin and streptomycin. The human breast cancer cell line MDA-MB-231 was purchased from China Infrastructure of Cell Line Resources (Peking Union). Mouse primary testis stromal cells (MTS) were isolated from immature mice as previously described ${ }^{23}$ and were maintained at $37^{\circ} \mathrm{C}$ and $5 \% \mathrm{CO}_{2}$ in DMEM supplemented with $10 \%$ FBS and $1 \%$ penicillin and streptomycin. Authentication of the MDA-MB-231 cell line was performed by short tandem repeat 
DNA profiling and compared with a reference database. All the cell lines used in this work were shown to be free of mycoplasma contamination. For the cytotoxicity assay, the cell lines were seeded into $96-w e l l$ plates at $37^{\circ} \mathrm{C}$ and sub-cultured for $24 \mathrm{~h}$. The cell cultured medium was subsequently replaced by fresh medium containing different 3-BP concentrations at different conditions. After further incubation for $24 \mathrm{~h}$, the cell viability was assessed using a CCK-8 Kit assay.

\subsection{Measurement of ATP levels}

Pan-02 pancreatic cancer cells $\left(1 \times 10^{6}\right)$ were seeded into 6-well plates and cultured at $37^{\circ} \mathrm{C}$ in $5 \% \mathrm{CO}_{2}$ for $24 \mathrm{~h}$. The medium was then replaced by a fresh medium containing 20 or $40 \mu \mathrm{M} 3-\mathrm{BP}$. After incubation at $37^{\circ} \mathrm{C}$ for 6 or $12 \mathrm{~h}$, the cultured cells were collected and ATP was measured using an Enhanced ATP Assay Kit (Beyotime: S0027 ) according to the manufacturer's instructions. For assessing intratumoral ATP levels, tumor tissues were harvested from pancreatic cancer tumor-bearing C57BL mice and analyzed according to the Enhanced ATP Assay Kit protocol.

\subsection{Animal experiments}

To evaluate the in vivo anti-tumor efficacy of T-Lipo-3BP, a mouse tumor model was established by inoculating $1 \times 10^{6}$ Pan-02 tumor cells into the right flank of C57BL/6 mice. The mice were divided into five groups, with six animals in each group. When the tumor volume reached approximately $150 \mathrm{~mm}^{3}$ (volume $=$ length $\times$ width2/2), different drug formulations were injected intravenously into the mice.

For in vivo imaging experiments, Pan-02 tumor-bearing nude mice were injected intravenously with T-Lipo-3BP-Cy7. After 2 and $24 \mathrm{~h}$, the biodistribution of nanoparticles was evaluated using a Maestro TM in vivo optical imaging system.

To assess the potential side effects of T-Lipo-3BP, C57BL mice bearing Pan-02 tumors were randomly divided into 5 groups: saline, 3-BP, T-Lipo-3BP, T-Lipo and Lipo-3BP. Meanwhile, the normal mice were used for healthy control. The mice were treated on three occasions at two day intervals. Two days after the final treatment, the mice were euthanized. Serum was collected for biochemical analysis and the major organs were resected for histopathology.

All animal procedures were approved by the Committee on the Ethics of Animal 
Experiments of the Health Science Center of Peking University (Beijing, China).

\subsection{Western blotting}

After treatment with varying drug concentrations, cells were harvested and lysed in RIPA buffer. The expression of MCT1 was subsequently analyzed by western blotting. Total protein $(20 \mu \mathrm{g})$ was separated on $10 \%$ polyacrylamide gels and proteins were transferred to a polyvinylidene fluoride (PVDF) membrane.

\subsection{Statistical analysis}

Statistical analysis was conducted using the student $t$-test for comparison of two groups and one-way ANOVA for multiple groups, followed by Newman-Keuls test. A $P$ value of less than 0.05 was considered significant.

\section{Results and Discussion}

\subsection{Preparation and characterization of T-Lipo-3BP nanoparticles}

To construct liposomal nanoparticles that target the tumor vasculature, we first conjugated the tumor targeting peptide CREKA to DSPE-PEG-MAL by the Michael addition reaction between the thiol of the peptide cysteine and the maleimide at the PEG terminus of the lipid monomer (Figure S1). The successful synthesis of DSPE-PEG-CREKA was confirmed using matrix-assisted laser desorption/ionization time-of-flight mass spectrometry (MALDI-TOF-MS) and nuclear magnetic resonance (NMR) analyses. The molecular weight of DSPE-PEG-MAL increased from 3800 to 4402 following peptide conjugation, as measured by MALDI-TOF-MS (Figure S2). Furthermore, the characteristic peaks of DSPE, PEG and MAL were clearly detected by NMR spectroscopy in the DSPE-PEG-MAL sample (Figure S3A), while the MAL peak disappeared following the CREKA conjugation (Figure S3B). The CREKA peptide coated liposome nanoparticles loaded with 3-bromopyruvate (3-BP) were subsequently prepared using a thin-film dispersion hydration method, as described by us previously [22]. To optimize the drug loading efficiency, three different mass ratios of lipid to 3-BP drug were tested (i.e. 20:15, 20:17 and 20:20). Drug encapsulation efficiency was highest (47.23\%) when the ratio of lipid to 3-BP was 20:17 (Table S1), and this ratio was chosen for subsequent studies. Transmission electron microscopy 
(TEM) showed that the empty (T-Lipo) and drug-loaded (T-Lipo-3BP) liposomal nanoparticles had a typical spherical structure (Figure 2A and 2C). Dynamic light scattering (DLS) analysis revealed a rather uniform size distribution for both nanoparticles, with a slight increase in the average hydrodynamic diameter from $141.5 \mathrm{~nm}$ for T-Lipo to $160.5 \mathrm{~nm}$ for T-Lipo-3BP (Figures 2B and 2D) and the PDI were 0.169 and 0.090 respectively.

We next investigated the 3-BP release profile of the liposomes in vitro by measuring the absorbance in the dialysate at $204 \mathrm{~nm}$ using high-performance liquid chromatography (HPLC). At pH 6.8, approximately $80 \%$ of the 3-BP was released from the T-Lipo-3BP nanoparticles within the first $30 \mathrm{~h}$ (Figure 2E), while less than $60 \%$ of drug was released over the same period at $\mathrm{pH}$ 7.4. Based on this $\mathrm{pH}$-dependent drug release pattern, an enhanced tumor specific release of 3-BP following binding of T-Lipo-3BP to fibrin-fibronectin complexes (microthrombi) on tumor vascular wall would be expected due to the low $\mathrm{pH}$ of the tumor microenvironment. In addition, the stability of T-Lipo-3BP was investigated by labeling the nanoparticles with membrane dye Dio (green) whose fluorescence is only detectable when inserted into an intact lipid layer. After incubating the nanoparticles in serum for $48 \mathrm{~h}$, there was a slight decrease (about 15\%) in fluorescent intensity (Figure 2F), demonstrating that the structural integrity of the particles was maintained.

\subsection{MCT1-dependent in vitro cytotoxicity of T-Lipo-3BP}

Monocarboxylate transporter 1 (MCT1), which is particularly highly expressed under hypoxic conditions, such as those found in the tumor microenvironment, is reported to mediate the cellular uptake of 3-BP in tumor cells [12-14]. To investigate the role of MCT1 in the uptake of T-Lipo-3BP-derived 3-BP, we first examined MCT1 expression in pancreatic cancer cells (Pan-02) and found that it was indeed more highly expressed under hypoxic conditions than in normoxia (Figure S4A). Consistent with this result, the viability of Pan- 02 cells was reduced by approximately $20 \%$ and $50 \%$ following treatment with T-Lipo-3BP containing $20 \mu \mathrm{M}$ or $40 \mu \mathrm{M}$ of 
3-BP, respectively for $24 \mathrm{~h}$, but only under hypoxic conditions (Figure 3A). The effect was stronger at $\mathrm{pH} 6.8$ than at $\mathrm{pH}$ 7.4. These data together demonstrate that the combination of hypoxia and lower $\mathrm{pH}$ led to the most cell killing.

To determine whether T-Lipo-3BP kills tumor cells in a MCT1 dependent manner, we investigated the effect of T-Lipo-3BP treatment on other two cell lines, the human breast cancer cell line MDA-MB-231, and mouse testis stromal cells (MTS), which have low ${ }^{6}$ (Figure S4B\&C), and negligible (Figure S4D) MCT1 expression, respectively. Cell viability assays showed that even at 3-BP concentrations in T-Lipo-3BP as high as $56 \mu \mathrm{M}, 100 \%$ of MDA-MB-231 and MTS cells remained viable after $24 \mathrm{~h}$ of treatment at $\mathrm{pH} 7.4$ under hypoxic conditions (Figure 3B,C). However, almost all Pan-02 cells were dead under the same conditions. Taken together, these data suggest that T-Lipo-3BP cytotoxicity is closely associated with the MCT1 expression levels in tumor cells and testis stromal cells.

We next examined whether T-Lipo-3BP killed MCT1-positive tumor cells by inhibition of aerobic glycolysis. An enhanced ATP assay revealed that, after treating Pan-02 cells with T-Lipo-3BP containing $20 \mu \mathrm{M}$ or $40 \mu \mathrm{M}$ of 3-BP for 6 or $12 \mathrm{~h}$, T-Lipo-3BP significantly reduced the cellular ATP content in a concentration and time-dependent manner (Figure 3D). This suggests that ATP production from aerobic glycolysis was effectively blocked by T-Lipo-3BP treatment, and that this was the likely mechanism of cell death.

\subsection{In vivo tumor targeting of T-Lipo-3BP}

The ability to target cytotoxic agents direct to tumors plays a decisive role in efficient cancer therapy. To investigate the tumor targeting capability of T-Lipo-3BP, we examined the biodistribution of Cy7-labeled nanoparticles after intravenous administration into Pan-02 tumor-bearing mice. Two hours after nanoparticle administration, in vivo imaging showed a weak fluorescence signal over much of the mouse body, but there was a very strong fluorescence signal at the site of the tumor (Figure 4A). After $24 \mathrm{~h}$, a strong fluorescence signal remained within the tumor region, but much less fluorescence was detected in other regions, confirming highly effective 
tumor targeting in vivo.

\subsection{In vivo anti-tumor activity of T-Lipo-3BP}

A prerequisite for in vivo anti-tumor activity of T-Lipo-3BP is that the target tumor expresses MCT1. We thus performed ex vivo immunohistochemistry of Pan-02 tumor tissue from our murine model and showed that the core of the tumors $\left(\sim 150 \mathrm{~mm}^{3}\right)$ had higher MCT1 expression, the same area where significant hypoxia-inducible factor $1 \alpha$ (HIF-1 $\alpha$ ) expression was observed (Figure S5). This confirmed that the central region of the tumors was highly anaerobic and that the model was an effective target for 3-BP. The Pan-02 pancreatic tumor-bearing mice were then injected intravenously with different formulations, including free 3-BP, targeted empty nanoparticles (T-Lipo), nontargeted drug-loaded nanoparticles (Lipo-3BP) or T-Lipo-3BP (3-BP), every two days for a total of 8 injections. The tumor volume was measured every 6 days using vernier calipers. Compared with saline and T-Lipo, the growth of tumors was significantly inhibited by 3-BP, Lipo-3BP or T-Lipo-3BP (Figure 4B). Clearly, T-Lipo-3BP at the same dosage outperformed free drug and Lipo-3BP, and dramatically reduced the tumor volume. Tumor mass was also assessed two days after the last treatment (Figure 4C). Mice injected with T-Lipo-3BP had a small tumor burden in contrast to the other groups, as demonstrated by a significant reduction in tumor weight. Together, these results demonstrate that T-Lipo-3BP effectively targets to tumors and inhibits their growth.

To examine the mechanism by which T-Lipo-3BP killed tumor cells, we evaluated apoptosis in the tumor tissue using terminal deoxynucleotidyl transferase-mediated deoxyuridine triphosphate nick-end labeling (TUNEL). TUNEL-positive cells were much more abundant in T-Lipo-3BP-treated tumors than in the other groups (Figure S6). We also observed that T-Lipo-3BP significantly reduced intratumoral ATP production (Figure 4D), while free 3-BP treatment only decreased the ATP level slightly.

\subsection{In vivo tolerability of T-Lipo-3BP}


The in vivo safety of T-Lipo-3BP was evaluated using normal C57BL mice. After intravenous administration of T-Lipo-3BP on three occasions at two day intervals, no loss of body weight was detected, with a similar result to the saline control group (Figure S7). We also performed histological examination of the major organs including the heart, liver, spleen, lung and kidney. Systemic administration of T-Lipo-3BP did not induce damage in any of the organs examined (Figure 5A), while administration of free 3-BP resulted in some adverse pathology in the liver. This is notable because serious liver burden is the dose-limiting side effect of free 3-BP in the clinical setting. We further investigated whether T-Lipo-3BP treatment resulted in functional abnormalities in the major organs of mice by assessing serum concentrations of biochemical indicators of liver, heart and renal function. Following three doses of T-Lipo-3BP, no significant changes in any of the indicators were observed compared with saline group. In contrast, free 3-BP significantly increased total bilirubin (TBIL; a well-known marker of liver cell damage) in the serum (Figures 5B and S8).

\section{Conclusions}

In summary, we have developed a tumor vascular endothelium targeted liposome nanoparticle (T-Lipo-3BP) to specifically deliver the aerobic glycolysis inhibitor 3-BP to MCT1-overexpressing tumors in mice. We chose liposomes as the controlled release system because its safety in clinical use has been well established. T-Lipo-3BP nanoparticles were able to efficiently and safely deliver 3-BP to the tumor following systemic administration and therefore represent a promising vehicle targeting the aerobic glycolysis pathway of tumor cells overexpressing MCT1. The lack of detectable severe side effects, especially the hepatotoxicity that is characteristic of 3-BP, marks a major improvement over the current free aerobic glycolysis inhibitors. We believe that this technique not only provides an effective strategy for the clinical application of 3-BP, but also could be applied for the delivery of other aerobic glycolysis targeting drugs to expand the utility of this therapeutic technology. 


\section{Supporting Information}

The Supporting Information is available free of charge on the ACS Publications website at DOI:

Drug encapsulation efficiencies; DSPE-PEG-CREKA conjugation; MALDI-TOF-MS analysis; NMR spectroscopy of DSPE-PEG-CREKA; MCT1 expression in Pan-02, MDA-MB-231 and MTS cells; Immunohistochemical staining of MCT1 and HIF1- $\alpha$; Tunnel immumohistochemical staining of Pan-02 tumor tissue; The body weight changes of mice; Biochemical indicators of kidney injury during different treatment.

\section{Competing financial interests}

The authors declare no competing financial interests.

\section{Acknowledgments}

This work was supported by the National Natural Science Foundation of China (31200752, 31470969 and 31300822), Academy of Medical Sciences-Newton Advanced Fellowship and the National Distinguished Young Scientist program (31325010).

\section{References}

1. Lis, P.; Dylag, M.; Niedzwiecka, K.; Ko, Y. H.; Pedersen, P. L.; Goffeau, A.; Ulaszewski, S. The HK2 Dependent "Warburg Effect" and Mitochondrial Oxidative Phosphorylation in Cancer: Targets for Effective Therapy with 3-Bromopyruvate. Molecules 2016, 21 (12), 1730.

2. Ganapathy-Kanniappan, S.; Geschwind, J. F. H. Tumor glycolysis as a target for cancer therapy: progress and prospects. Mol. Cancer 2013, 12(1), 152.

3. Hanahan, D.; Weinberg, R. A. Hallmarks of cancer: the next generation. Cell 2011, 144 (5), 646-74. 
4. Vander Heiden, M. G.; Cantley, L. C.; Thompson, C. B. Understanding the Warburg effect: the metabolic requirements of cell proliferation. Science 2009, 324 (5930), 1029-33.

5. Pavlova, N. N.; Thompson, C. B. The emerging hallmarks of cancer metabolism. Cell. Metab. 2016, 23(1), 27-47.

6. Birsoy, K.; Wang, T.; Possemato, R.; Yilmaz, O. H.; Koch, C. E.; Chen, W. W.; Brummelkamp, T. R. MCT1-mediated transport of a toxic molecule is an effective strategy for targeting glycolytic tumors. Nat. Genet. 2013, 45(1), 104-108.

7. Sprowl-Tanio, S.; Habowski, A. N.; Pate, K. T.; McQuade, M. M.; Wang, K.; Edwards, R. A.; Grun, F.; Lyou, Y.; Waterman, M. L. Lactate/pyruvate transporter MCT-1 is a direct Wnt target that confers sensitivity to 3-bromopyruvate in colon cancer. Cancer Metab. 2016, 4(1), 20.

8. Marrache, S.; Dhar, S. The energy blocker inside the power house: Mitochondria targeted delivery of 3-bromopyruvate. Chem. Sci. 2015, 6 (3), 1832-1845.

9. Ko, Y. H.; Smith, B. L.; Wang, Y.; Pomper, M. G.; Rini, D. A.; Torbenson, M. S.; Hullihen, J.; Pedersen, P. L. Advanced cancers: eradication in all cases using 3-bromopyruvate therapy to deplete ATP. Biochem. Bioph. Res. Co. 2004, 324 (1), 269-75.

10. Wu, L.; Xu, J.; Yuan, W.; Wu, B.; Wang, H.; Liu, G.; Cai, S. The reversal effects of 3-bromopyruvate on multidrug resistance in vitro and in vivo derived from human breast MCF-7/ADR cells. Plos One 2014, 9(11), e112132.

11. Ganapathy-Kanniappan, S.; Vali, M.; Kunjithapatham, R.; Buijs, M.; Syed, L. H.; Rao, P. P.; Geschwind, J. F. 3-bromopyruvate: a new targeted antiglycolytic agent and a promise for cancer therapy. Curr. Pharm. Biotechno. 2010, 11(5), 510-517.

12. Allen, E.; Miéville, P.; Warren, C. M.; Saghafinia, S.; Li, L.; Peng, M. W. .; Hanahan, D. Metabolic symbiosis enables adaptive resistance to anti-angiogenic therapy that is dependent on mTOR signaling. Cell Rep. 2016, 15(6), 1144-1160.

13. Azevedo-Silva, J.; Queirós, O.; Ribeiro, A.; Baltazar, F.; Young, K. H.; Pedersen, P. L.; Casal, M. The cytotoxicity of 3-bromopyruvate in breast cancer cells 
depends on extracellular pH. Biochem. J. 2015, 467(2), 247-258.

14. Miranda-Gonçalves, V.; Granja, S.;Martinho, O.; Honavar, M.; Pojo, M.; Costa, B. M.; Costa, P. Hypoxia-mediated upregulation of MCT1 expression supports the glycolytic phenotype of glioblastomas. Oncotarget 2016, 7(29), 46335-46353.

15. Ko, Y. H.; Verhoeven, H. A.; Lee, M. J.; Corbin, D. J.; Vogl, T. J.; Pedersen, P. L. A translational study "case report" on the small molecule "energy blocker" 3-bromopyruvate (3BP) as a potent anticancer agent: from bench side to bedside. J. Bioenergy. Biomembr. 2012, 44 (1), 163-70.

16. Kraft, J. C.; Freeling, J. P.; Wang, Z.; Ho, R. J. Emerging research and clinical development trends of liposome and lipid nanoparticle drug delivery systems. J. Pharm. Sci. 2014, 103(1), 29-52.

17. Meng, H.; Wang, M.; Liu, H.; Liu, X.; Situ, A.; Wu, B.; Nel, A. E. Use of a lipid-coated mesoporous silica nanoparticle platform for synergistic gemcitabine and paclitaxel delivery to human pancreatic cancer in mice. ACS nano 2015, 9(4), $3540-3557$.

18. Akbarzadeh, A.; Rezaei-Sadabady, R.; Davaran, S.; Joo, S. W.; Zarghami, N.; Hanifehpour, Y.; Nejati-Koshki, K. Liposome: classification, preparation, and applications. Nanoscale Res. Lett. 2013, 8(1), 102.

19. Li, F.; Zhao, X.; Wang, H.; Zhao, R.; Ji, T.; Ren, H.; Anderson, G. J.; Nie, G.; Hao, J. Multiple Layer-by-Layer Lipid-Polymer Hybrid Nanoparticles for Improved FOLFIRINOX Chemotherapy in Pancreatic Tumor Models. Adv. Funct. Mater. 2015, 25 (5), 788-798.

20. Agemy, L.; Sugahara, K. N.; Kotamraju, V. R.; Gujraty, K.; Girard, O. M.; Kono, Y.; Mattrey, R. F.; Park, J. H.; Sailor, M. J.; Jimenez, A. I.; Cativiela, C.; Zanuy, D.; Sayago, F. J.; Aleman, C.; Nussinov, R.; Ruoslahti, E. Nanoparticle-induced vascular blockade in human prostate cancer. Blood 2010, 116 (15), 2847-56.

21. Simberg, D.; Duza, T.; Park, J. H.; Essler, M.; Pilch, J.; Zhang, L.; Derfus, A. M.; Yang, M.; Hoffman, R. M.; Bhatia, S.; Sailor, M. J.; Ruoslahti, E. Biomimetic amplification of nanoparticle homing to tumors. P. Natl. Acad. Sci. USA. 2007, $104(3), 932-6$. 
22. Zhang, Y.; Wei, J.; Liu, S.; Wang, J.; Han, X.; Qin, H.; Lang, J.; Cheng, K.; Li, Y.; Qi, Y.; Anderson, G. J.; Sukumar, S.; Li, S.; Nie, G. Inhibition of platelet function using liposomal nanoparticles blocks tumor metastasis. Theranostics 2017, 7 (5), 1062-1071.

23. Guan, K.; Wolf, F.; Becker, A.; Engel, W.; Nayernia, K.; Hasenfuss, G. Isolation and cultivation of stem cells from adult mouse testes. Nat. Protoc, 2009, 4(2), 143-154.

A

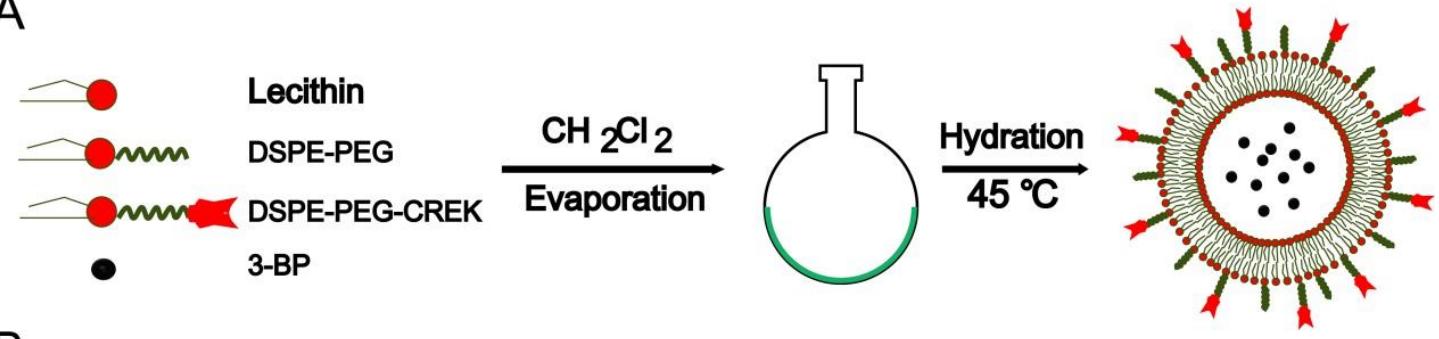

B

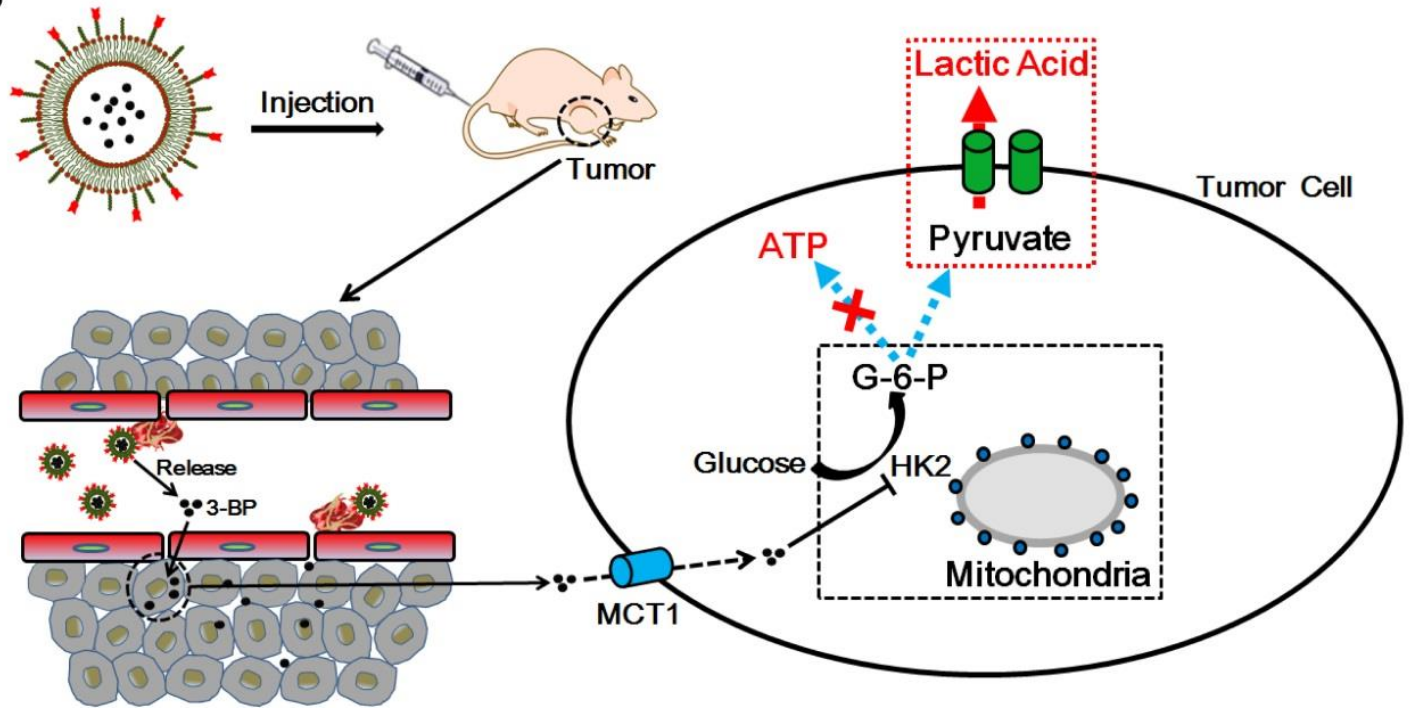

Figure 1. The construction of T-Lipo-3BP nanoparticles and the proposed mechanism of action in tumor vessels. (A) Schematic illustration of synthesis of the T-Lipo-3BP nanoparticles and the encapsulation of the glycolysis inhibitor 3-BP. (B) After intravenous administration of T-Lipo-3BP into tumor-bearing mice, T-Lipo-3BP targets to the tumor vascular wall and locally releases 3-BP. The released drug is then delivered into tumor cells via the transporter MCT1, and subsequently inhibits hexokinase-2 (HK-2). This in turn blocks ATP supply for tumor cells without 
affecting normal cells.

A

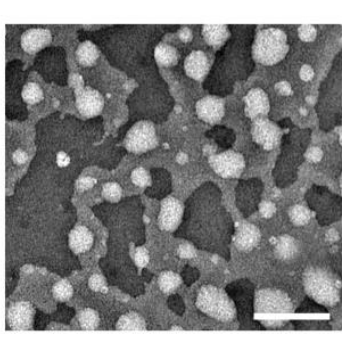

C

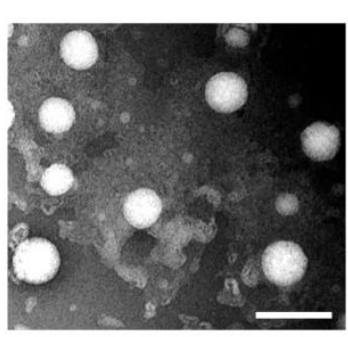

B
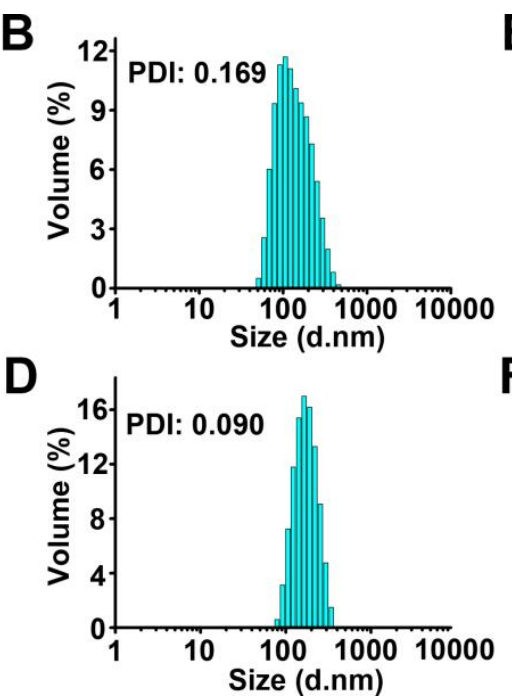

$\mathbf{F}$
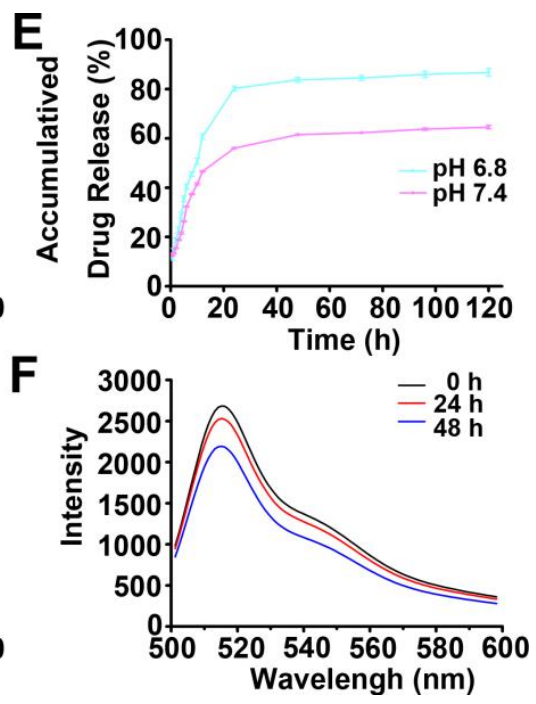

Figure 2. Characterization of T-Lipo-3BP nanoparticles. (A,C) TEM images of representative empty (A) and drug-loaded (C) liposomal nanoparticles. Scale bars are $200 \mathrm{~nm}$. (B,D) DLS analysis showing the size distribution of empty (B) and drug-loaded (D) liposomal nanoparticles. (E) In vitro release kinetics of 3-BP from T-Lipo-3BP at different $\mathrm{pH}$ values $(\mathrm{pH} 6.8$ is characteristic of the tumor microenvironment; $\mathrm{pH} 7.4$ represents physiological conditions in the blood). Data represent the mean \pm s.d. of three independent experiments. (F) In vitro stability of Dio-labeled T-Lipo-3BP. The nanoparticles are quite stable over time in serum at pH7.4. Representative results of three independent experiments are shown. 

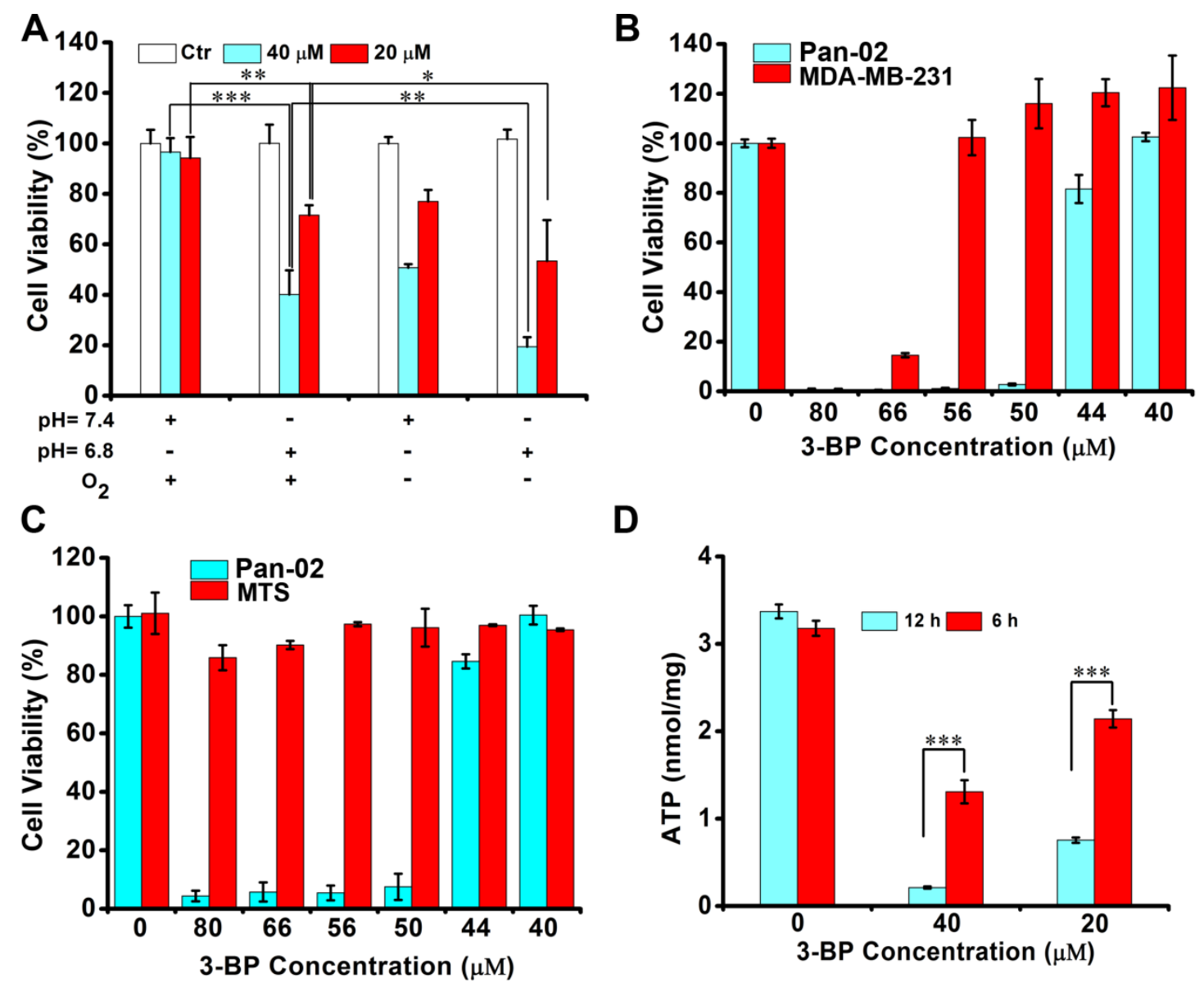

Figure 3. Effects of T-Lipo-3BP on cell viability and ATP production. (A) Viability of Pan-02 tumor cells after treatment with T-Lipo-3BP containing $40 \mu \mathrm{M}$ or $20 \mu \mathrm{M}$ 3-BP under both normoxic and hypoxic conditions at $\mathrm{pH} 7.4$ and 6.8 at $37^{\circ} \mathrm{C}$ for $24 \mathrm{~h}$. ${ }^{*} p<0.05, * * p<0.01, * * * p<0.001$. Error bars represent the mean \pm s.d. of three independent experiments. (B) Cytotoxicity of T-Lipo-3BP contaning different concentrations of 3-BP in Pan-02 and MDA-MB-231 tumor cell lines after $24 \mathrm{~h}$ of treatment at $\mathrm{pH} 7.4$ under hypoxic conditions. (C) Cytotoxicity of T-Lipo-3BP containing different concentrations of 3-BP in Pan-02 tumor cells and MTS cells after $24 \mathrm{~h}$ of treatment at $\mathrm{pH} 7.4$ under hypoxic conditions. (D) Cellular levels of ATP after treatment of Pan-02 cells with different concentrations of 3-BP in T-Lipo-3BP. Error bars represent the mean \pm s.d. of three independent experiments, $* * * p<0.001$. 

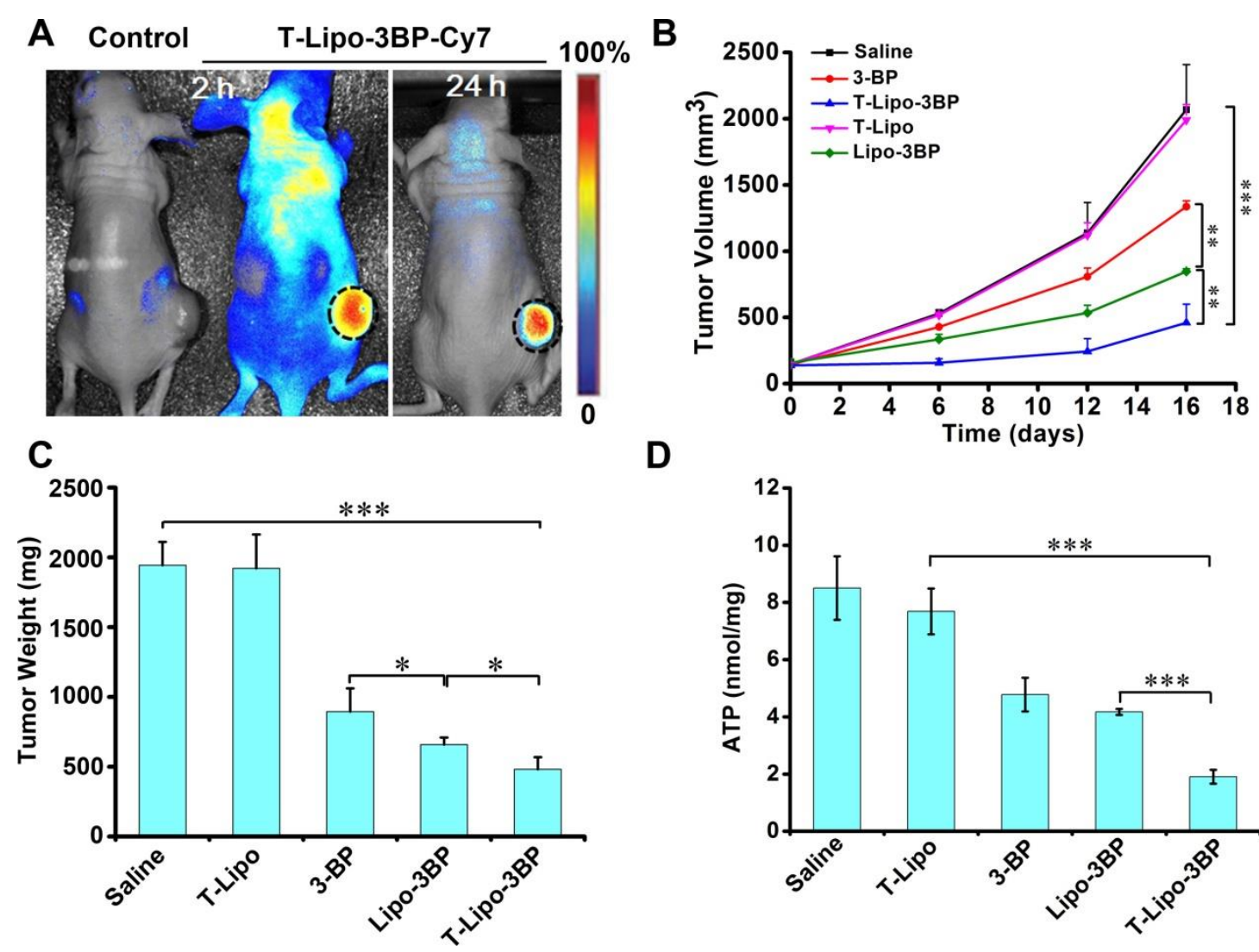

D

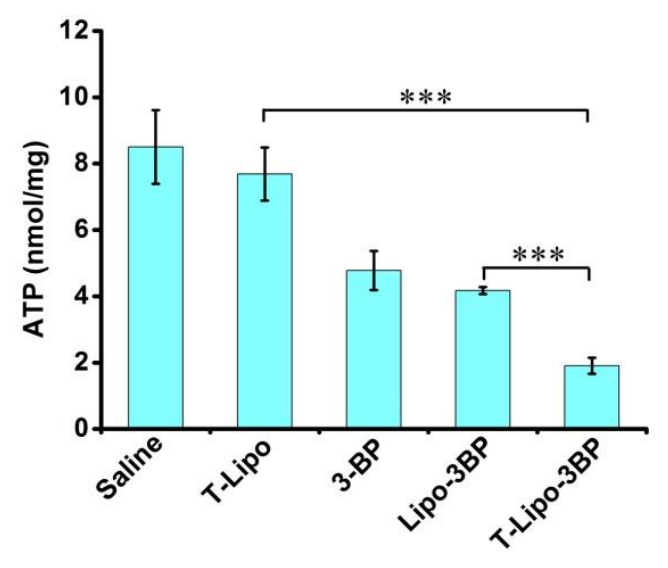

Figure 4. In vivo tumor targeting and anti-tumor activity of T-Lipo-3BP. (A) In vivo imaging of Pan-02 tumor-bearing mice 2 and $24 \mathrm{~h}$ after administration of Cy7-labeled T-Lipo-3BP. Black circles indicate the site of the tumors. $(n=3)$. (B) The size of the tumors was recorded at different time points after intravenous administration of various drug formulations. Data are presented as mean \pm s.d. $(n=6)$. (C) Tumors were harvested two days after the last treatment and weighed. Data are presented as the mean \pm s.d. $(n=3)$. (D) Intratumoral ATP levels were measured in tumors harvested two days after the last treatment with different drug formulations. Data are presented as the mean \pm s.d. $(\mathrm{n}=3) .{ }^{*} p<0.05, * * p<0.01, * * * p<0.001$. 
A
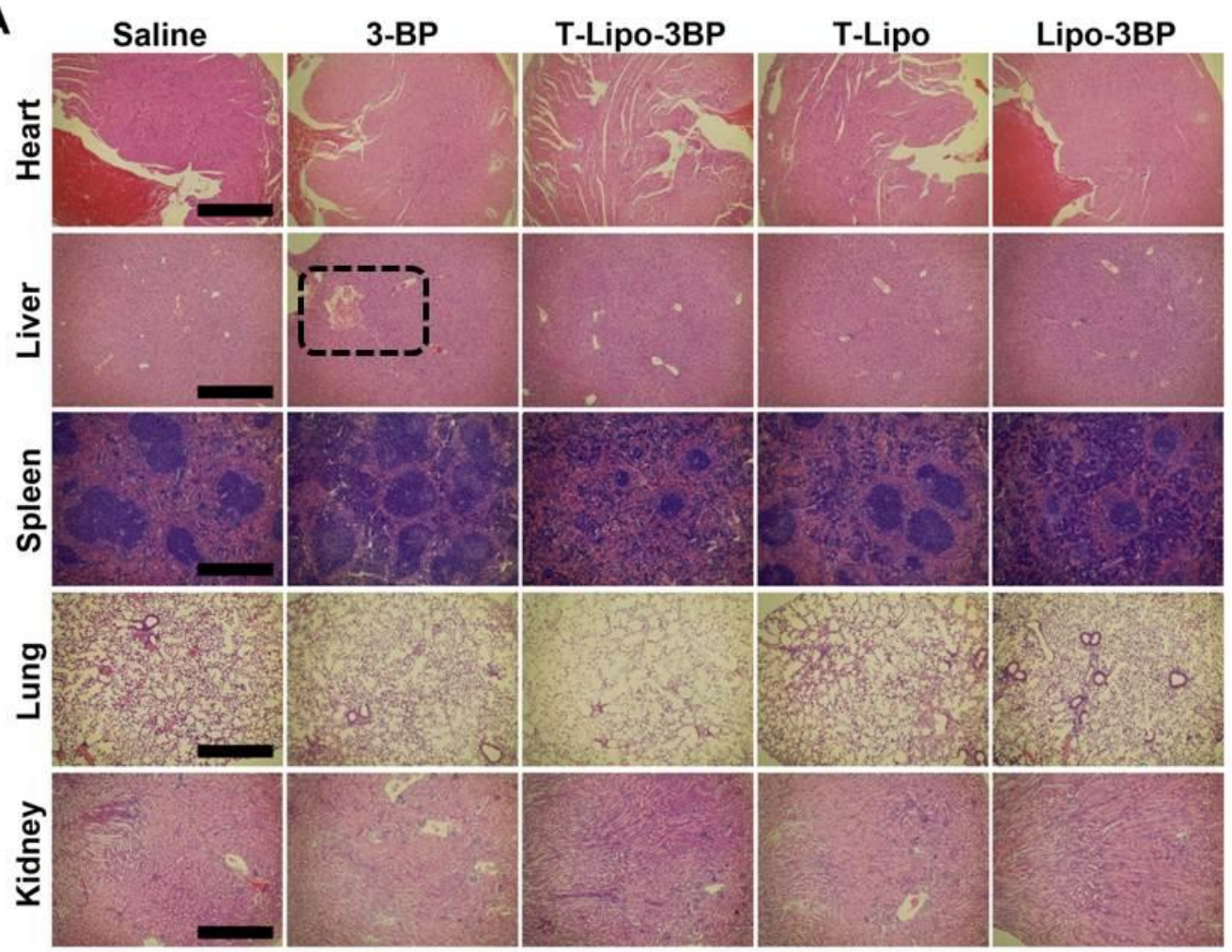

B
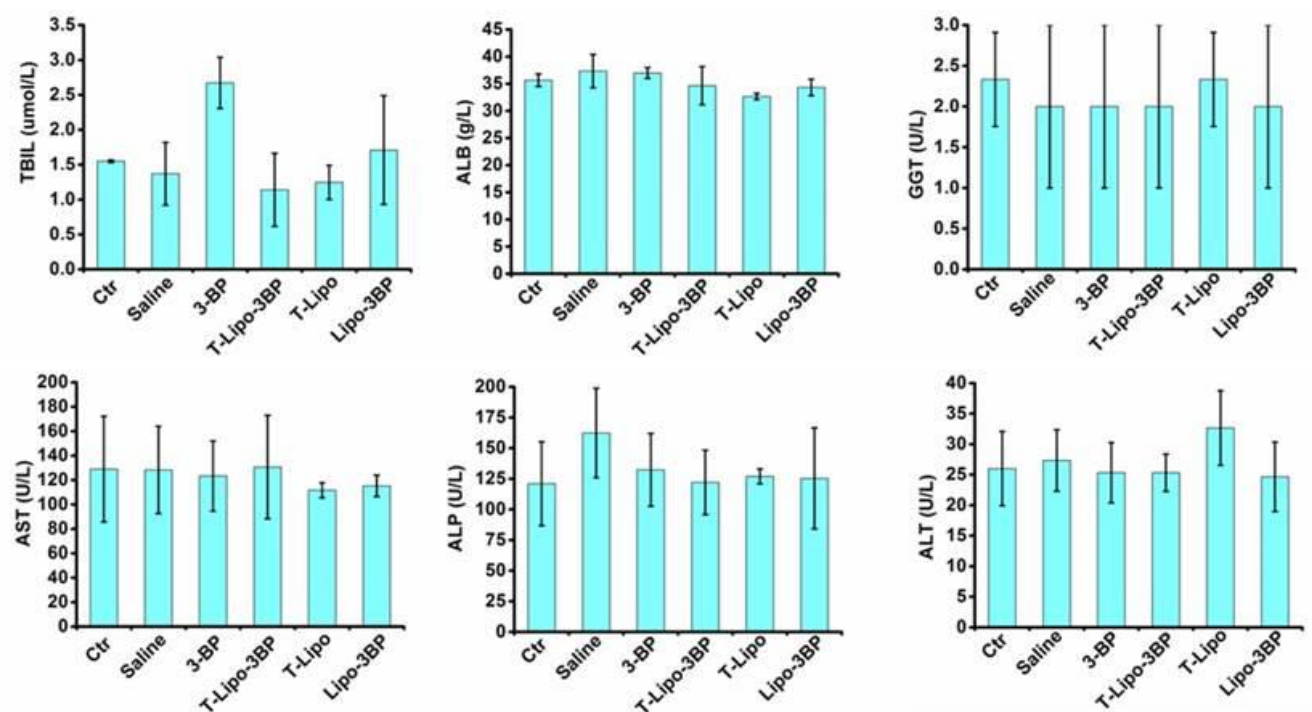

Figure 5. Safety evaluation of T-Lipo-3BP in vivo. (A) H\&E staining of major organs removed two days following the final treatment with various drug formulations. Some hepatic damage (indicated by the dotted line) was apparent in the group treated with free 3-BP. The images are representative of three independent experiments. (B) After intravenous administration of various drug formulations, whole blood was drawn from retro-orbital venous plexus of wild-type C57BL mice and serum was analyses for various markers of tissue injury. Data represent the mean \pm s.d. $(n=6)$. 


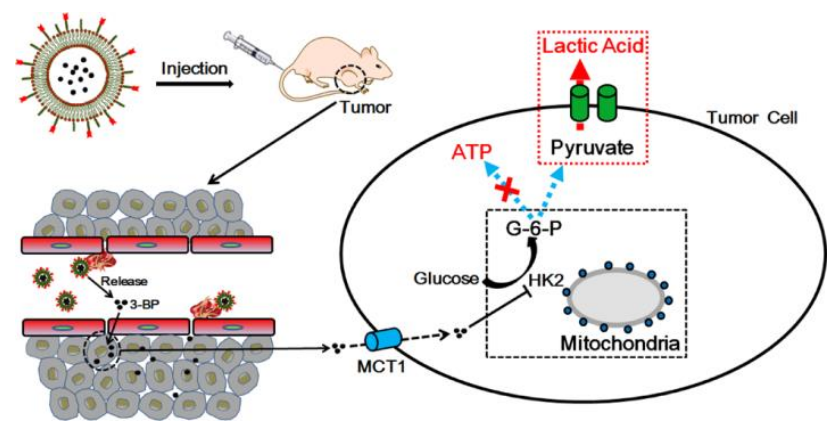

For Table of Contents Only 